\title{
Linguistique et science de la littérature
}

\author{
François Rastier \\ ER-TIM, Institut National des Langues Orientales, Paris*
}

La tradition grammaticale n'a pas cherché à décrire la littérature. Toutefois, depuis la formation de la linguistique voici deux siècles, science du langage et science de la littérature voisinent au sein des sciences de la culture. Cela a été occulté par les grammaires formelles et le cognitivisme orthodoxe. Développant la méthode comparatiste, le structuralisme a cependant permis un progrès théorique exploité par la sémiotique des cultures. Au-delà de la linguistique des textes, il reste à concevoir et à faire admettre une linguistique des œuvres (ou opératique). La linguistique et les études littéraires ont en effet beaucoup à apprendre de leurs interactions.

Mots-clés : linguistique comparée, littérature, structuralisme, grammaire, sémiosis, sémiotique des cultures.

\section{Limites de la problématique logico-grammaticale}

À sa fondation intellectuelle par l'école d'Alexandrie, la grammaire était une discipline auxiliaire pour l'entreprise philologique de constitution de corpus littéraires et notamment, en premier lieu, du corpus homérique. Issu de cette « école », Denys le Thrace est reconnu comme l'auteur du premier traité de grammaire qui nous soit parvenu et où figurent les catégories fondamentales de notre tradition, notamment les parties du discours dont l'ordre et le nombre sont restés invariables, jusqu'à Chomsky inclus. À la fin de son traité, il indique que la plus belle partie de la grammaire est la critique des œuvres, critique tout à la fois philologique et esthétique.

Cette dimension restera hélas négligée dans la tradition scolaire qui s'ouvre alors, car la grammaire devient une discipline propédeutique, la première du trivium, enseignée aux jeunes enfants ${ }^{1}$. Les questions esthétiques n'étaient développées qu'à la fin du cycle d'études, avec la classe de rhétorique. Il ne s'agissait plus

\footnotetext{
*frastier@gmail.com

${ }^{1}$ L'iconographie les montre à l'occasion menacés des verges, voire fessés publiquement, comme c'est le cas dans l'église Saint Augustin de San Giminiano. Souvent, la grammaire est figurée comme une institutrice rébarbative et fouettarde.
} 
d'apprendre des règles valables partout, pour former les phrases correctes d'un langage ordinaire que l'on nommait le " langage pédestre » (sermo pedestris), mais au contraire de déceler et caractériser des œuvres singulières témoignant d'un exemplaire « langage ailé » (sermo alatus).

En somme, jusqu'à la formation de la linguistique, les réflexions sur le langage (au-delà même de l'Occident) se sont divisées en trois parties : la grammaire, scolaire et centrée sur une langue de culture et/ou religieuse : sanscrit, arabe coranique, grec classique homérique, latin ; une logique philosophique, dont témoignent notamment les grands traités scolastiques sur le langage et l'esprit ; une herméneutique littéraire ou religieuse à fonction éducative, voire édifiante, depuis les interprétations stoïciennes d'Homère.

Appuyés sur une critique de l'ontologie, les théories rhétoriques d'humanistes comme Lorenzo Valla, Luis Vivès, Érasme, Giordano Bruno auraient permis un changement radical de problématique. Ils ont donc été marginalisés par la tradition universitaire, qui admettait mal la critique radicale de la conception logique du langage partagée par les grammairiens et les dialecticiens.

Traditionnellement dominante dans les sciences du langage, la problématique logico-grammaticale persiste à entraver la compréhension des rapports entre linguistique et littérature. La grammaire a toujours privilégié les règles et s'est identifiée avec leur recherche, leur formulation et leur articulation, jusqu'à postuler qu'il existe un seul système de la langue. Une approche polysystématique semble préférable : les régularités observées dans certains domaines de structuration, comme la syllabe ou le syntagme, ne font pas système, car nous avons affaire à différentes échelles et degrés d'organisation (l'auteur, 1987 : ch. $3)$. Quelles que soient les politiques d'optimisation, les interactions entre systèmes locaux déterminent en partie pour les conditions internes de leur évolution, les déterminations externes venant d'autres institutions sociales que la langue.

En outre, la grammaire vise pour ainsi dire au sans-faute : toute phrase française se doit d'obéir à ses règles et cette docilité lui permet d'être reconnue comme telle, faute de quoi elle serait exclue comme agrammaticale. Souvent même, comme on l'a vu avec Chomsky, Saumjan ou Montague, les grammaires se voudraient universelles, exprimant les règles de l'esprit humain, comme l'autorise la facile syllepse entre le logos comme enchaînement rationnel, dont dérive le nom même de la logique, et le logos comme discours ou chaîne linguistique. Les stoïciens ont bien certes distingué le discours interne, celui de la rationalité (logos endiathétos) et le discours externe (logos prophorikos) ; mais les deux acceptions renvoient l'une à l'autre, si bien que le préfixe -logie désigne encore un discours de connaissance (météorologie, pharmacologie, etc.).

Pour formuler des règles, la grammaire privilégie les formes d'organisation les plus récurrentes et dont la distribution est uniforme, bref celles qui 
constituent le « fond » de l'organisation structurelle du matériau linguistique. En revanche, les formes complexes, qui se profilent sur ce fond morphosyntaxique, lui échappent par leur complexité même, car elles ne sont saisissables par aucune de ses catégories, ni même peut-être par une pensée simplement catégorisante : n'étant pas discrètes, elles échappent à l'apodictique de ce que Jean-Claude Milner nommait le « différentiel syntaxique », bref à l'opposition entre grammatical et agrammatical.

On ne saurait cependant en tenir rigueur à la grammaire, car elle assume ces limites et la description des formes textuelles et de leurs évolutions n'est pas son objectif. En revanche, la linguistique des textes et les études littéraires partagent l'objectif, au sein de corpus définis, de différencier les textes entre eux, et, au sein des textes, de caractériser les formes sémantiques et expressives, en décrivant leur évolution.

\section{Formation de la linguistique et édification des sciences de la culture}

Avant la formation de la linguistique, les grammairiens ne dédaignaient pas les remarques sur les œuvres littéraires et recommandaient les bons auteurs ; pour leur part, les écrivains étaient tous formés aux langues anciennes, si bien que la connaissance des langues et celle des œuvres allaient de pair au sein des Lettres.

Ces relations se développèrent à une toute autre échelle avec les Lumières et la formation des sciences de la culture à la fin du XVIII siècle, lors de la création de la linguistique comparée et de la littérature comparée. Linguistes et philologues s'attachaient à établir des corpus écrits, notamment littéraires, comme celui des troubadours, reconstitué et édité par les romanistes allemands, les frères Schlegel en premier lieu.

La linguistique dépassait dès lors les vertueuses simplifications didactiques de la grammaire scolaire. Alors que la grammaire s'était développée pendant vingt siècles, la linguistique comparée renouvela le statut des réflexions sur le langage et les langues. La grammaire s'étendit à la grammaire comparée. La littérature ne se limita plus à proposer les exemples des bons auteurs et c'est une science de la littérature qui s'ébauche avec la littérature comparée. Linguistique comparée et littérature comparée voisinent au sein du continent nouveau qui se constitue avec l'édification des sciences de la culture, projet que l'on doit pour l'essentiel aux Lumières. L'étude des langues peut devenir une discipline noblement auxiliaire de l'étude des textes, œuvres littéraires comprises. Jusqu'à Saussure et au-delà, les études de langue et de littérature iront de pair, tant pour la constitution que pour l'interprétation des corpus. En outre, la linguistique his- 
torique et la littérature comparée ont su partager les exigences qu'impose leur méthodologie critique.

Cette situation nouvelle a cependant été occultée de diverses manières, tantôt par une conception mystique du langage, tantôt par une réduction de la linguistique à la grammaire. La mystique romantique du langage, qui doit beaucoup aux spéculations théosophiques d'auteurs comme Jacob Böhme, a accrédité l'idée que la littérature, et surtout la poésie, était une langue transcendante, écho du langage divin. Cette croyance, que l'on trouve de Hölderlin à Novalis, s'est perpétuée dans le romantisme tardif, dans certains cercles de poétique russe comme dans l'idéalisme de Croce qui inspire pour une part Coseriu, voire dans les dernières œuvres de Meschonnic, traversées par un souffle biblique. Pour Heidegger, la poésie témoigne des dieux disparus et c'est par elle que se révèle leur langage - qui se résume en fait à la langue allemande archaïque, purifiée et idéalisée. Ainsi, le romantisme tardif dont nous sommes encore tributaires a pu opposer les sciences et les arts, en minimisant le projet de connaissance des premières et en privilégiant les effusions sentimentales de l'Einfühlung littéraire, aujourd'hui prolongées dans certaines esthétiques de la réception. Dans le domaine des études littéraires, le courant déconstructeur a voulu récuser toute approche scientifique, voire simplement objectivante.

Un autre malentendu entre linguistique et études littéraires a voulu réduire la linguistique à la grammaire, comme on le fait trop souvent. Ainsi depuis plus d'un demi-siècle, les grammaires universelles et la linguistique cognitive ont rompu tout lien avec les corpus littéraires. Avec des formalismes nouveaux, elles ont repris les principes des grammaires générales qui précédaient la formation de la linguistique (voir Chomsky, Cartesian Linguistics, 1966). Les langues ne sont plus l'objet de la linguistique, c'est la grammaire (cf. Chomsky, 1984). Les textes, et a fortiori la littérature, disparaissent des préoccupations des linguistes comme de l'objet même de la linguistique. Avec les grammaires universelles contemporaines, le rejet des exemples littéraires s'est accru, au profit d'exemples aussi prosaïques que forgés. De plus, comme la grammaire, la philosophie du langage s'en est tenue au langage «pédestre » des scolastiques, jusqu'à Austin qui jugeait la poésie " pas très sérieuse » ou Strawson qui rejetait les poèmes homériques comme « oiseux » (spurious). Ainsi, la prévalence de la grammaire sur toutes les autres disciplines traitant des langues a conduit à la séparation entre linguistique et philologie ${ }^{2}$ voire à la disparition de cette dernière (c'est le cas en France où les formations de linguistique et de philologie ont disparu).

Comme la linguistique comparée travaille sur des corpus, elle conserve un lien avec la philologie qui les établit, lien si nécessaire que l'on a constitué récem-

\footnotetext{
2 Déjà, selon Raoul de La Grasserie, la philologie étudie la langue littéraire et la linguistique les langues (cf. De la classification objective et subjective des arts, des sciences et des lettres, Paris, Alcan, 1893).
} 
ment une philologie numérique. Pour la linguistique comparée, la séparation de fait entre linguistique et littérature n'a rien de nécessaire ni d'irréversible. Louis Havet déclarait par exemple en 1922 : « En fait les lettres (sous l'aspect, il est vrai, qui est le moins familier au vulgaire), sont matière à science au sens propre. Ce qui définit la science, c'est la logique, c'est la méthode ; ce n'est pas l'objet » (1922: p. 4).

Le structuralisme saussurien est une autoréflexion de la linguistique historique et comparée. Les différents foyers structuralistes (Moscou, Leningrad, Prague, Copenhague, Paris) ont toujours privilégié les textes. C'est l'usage constant dans tous les cercles structuralistes, des formalistes russes (issus du cercle de poétique de Moscou), au cercle de Prague (cf. Mukarovsky). Tous les grands linguistes travaillent sur des textes littéraires, de Saussure sur les Nibelungen et les poèmes homériques notamment, jusqu'à Jakobson sur un cycle épique russe.

Parallèlement, Cassirer part des sciences de la culture pour proposer une définition morphologique de la structure et, après étude de la linguistique historique et comparative (au tome premier de la Philosophie des formes symboliques), en vient à une théorie des formes symboliques (institutions comme le langage ou l'art ou le droit) qui rencontre la sémiologie générale de Saussure.

Ainsi, depuis l'entre-deux-guerres, maintes études linguistiques de premier plan ont pris pour objet la littérature, tout comme dans les années 1950-1970 les études se réclamant du structuralisme. À présent, la linguistique de corpus et les humanités numériques renouent des liens avec la philologie comme avec l'herméneutique matérielle.

La linguistique ne postule aucune exceptionnalité de la littérature, mais reconnaît une diversité qu'elle prend pour objet. Chaque discours définit de fait ses normes et les règles de la langue sont simplement des normes transdiscursives. Il serait donc illusoire de définir la littérature comme un écart par rapport à un langage ordinaire - qui à mes yeux du moins n'est pas moins mystérieux qu'elle. Le langage ordinaire se divise aussi en paliers normatifs, en genres et en discours : la science comme la littérature ont aussi leur « langage ordinaire », jargon infesté de stéréotypes, celui de la science normale et des best-sellers pour salle de transit.

Les différences entre discours et entre textes engagent à mieux préciser le rapport entre la règle et l'exception, en le reconduisant à la dualité saussurienne entre langue et parole. La règle se résume une régularité fréquente dans les usages de la langue à un moment historique donné : c'est une norme dominante. Comme elle ne s'applique jamais en tout lieu, l'exception décèle la norme, sans pourtant l'invalider, et elle ne la confirme qu'a contrario, tout en contribuant à son évolution.

Le langage littéraire ne transgresse les règles que d'un point de vue grammatical normatif. Du point de vue englobant de la linguistique descriptive, il n'est 
qu'un usage qui définit un discours et son élaboration continue n'a rien d'une transgression, quoiqu'ait prétendu certain satanisme post-romantique. Tout usage de la langue la modifie en effet potentiellement.

Au demeurant, le discours scientifique ne transforme pas moins les habitudes, en instaurant ses normes ; mais par rapport à d'autres discours, religieux, juridique, scientifique, etc., la littérature a la caractéristique de jouer avec ses propres normes, à les problématiser en les variant de manière critique. C'est pourquoi l'on peut caractériser des styles d'auteur, qui seraient des normes paradoxalement " idiolectales », et même des styles d'œuvre qui témoignent de projets artistiques singuliers. L'écrivain crée ainsi sa langue, ou plutôt concrétise à chaque œuvre un état possible de la langue. Édouard Glissant se fixait par exemple ces tâches : " établir la liste de tant de mots en nous dont le sens nous échappe, ou plus loin fixer la syntaxe de ce que nous balbutions » (cf. 1975 : p. 231).

En réfléchissant de manière critique l'usage de la langue, la création dans les arts du langage précède, avec de tout autres moyens et objectifs, la réflexion critique qui préside à la description scientifique. Mais reconnaître cela n'est pas plaider pour une connaissance poétique ou, pire encore, pour une poétisation de la science.

\section{3. Étude des æuvres et éléments d'opératique}

Révision. - En retour s'impose une révision, voire une refondation de la grammaire : non plus sur des catégories (au demeurant ethnocentriques) comme les « parties du discours» ou parts of speech partout invoquées dans les traitements automatiques du langage et dans les ontologies comme le «Web sémantique », mais sur des unités fondamentales découvertes jadis par la linguistique, comme le morphème ou le phonème ; sans oublier l'essentiel : les formes sémantiques, comme les thèmes, ou expressives, comme les courbes intonatives. Les relations syntaxiques même doivent être redéfinies, comme l'a fait Louis Tesnière avec la théorie de la translation. Par exemple, la notion de rection devient problématique : le verbe n'est plus le « roi » de la phrase susceptible de la régir; et que faire dans les langues sans opposition verbo-nominale?

Tout mot, toute phrase est un fragment de texte et la linguistique de corpus permet à présent, non pas de valider les grammaires formelles, mais de déceler leur peu d'utilité. Ainsi, dans les traitements automatiques du langage, l'échec cuisant du chomskysme, fondé sur des formalismes déterministes et ignorant les concepts même de texte et de corpus - d'où la déclaration de Chomsky, dès 1999, que « la linguistique de corpus n'existe pas ». Pour répondre aux demandes sociales, des formalismes tout autres, probabilistes, non seulement fréquentistes, mais bayésiens, se sont imposés avec l'intelligence artificielle de tradition connexionniste. 
Genèses. - La littérature et les arts du langage tirent parti du matériau linguistique pour innover sans cesse, créer des formes inouïes, qui souvent mettent en œuvre une pluralité sémiotique, comme le chant et la calligraphie pour la poésie.

La genèse continue des signes perpétue celle des langues. Comme l'homme n'a pas de langage inné, les langues sont nos œuvres et les arts du langage les perpétuent en les réélaborant. Les expressions formulaires, comme les expressions en quatre caractères du chinois, réfèrent à des œuvres antiques passées ainsi dans la langue. Les signes sont des fragments de textes, voire des passages d'œuvres ou des traces de mythes. Parmi tous ceux qui se créent chaque jour ne demeurent que ceux qui apparaissent dans des textes oraux ou écrits jugés mémorables.

En bref, les langues sont des œuvres à partir desquelles nous créons d'autres œuvres. La conscience de l'exemplarité des œuvres semble fort partagée : textes religieux, mythiques, poétiques, sont valorisés, mémorisés, transmis. De nos jours, la conception opératique $e^{3}$ des langues a été négligée, en raison des spéculations néo-darwiniennes sur l'origine du langage, qui est un tout autre problème, faussé au demeurant.

Le « matériau » linguistique n'est pas neutre ni uniforme : c'est un champ saturé dans lequel l'artiste provoque, exploite et réfléchit des prises de forme $\mathrm{e}^{4}$. En somme, la genèse des signes par la sémiosis particulière des textes poursuit et concrétise celle de la langue. Comme elle relève d'une praxéologie, cette dimension n'a pas été réfléchie par la tradition grammaticale et la rémanence des ontologies philosophiques en linguistique contemporaine n'a guère permis d'aller plus loin. La pragmatique s'est certes préoccupée de ce que l'on fait avec la langue, mais non de comment on la fait.

Avec des objectifs et des méthodes différents, l'art du langage et la science du langage élaborent des points de vue complémentaires, dont témoignent les emprunts réciproques des linguistes et des analystes et critiques de la littérature. Au-delà, les linguistes trouvent dans la littérature de nouveaux observables et une critique silencieuse de leurs schématisations trop simples : à eux de relever ce défi et de savoir apprendre auprès des œuvres.

Complémentairement, bien des écrivains, de Stéphane Mallarmé à Italo Calvino et Claude Simon témoignent d'une connaissance théorique qui se reflète dans leur pratique créatrice. Les traducteurs littéraires, tout à la fois linguistes et créateurs, concrétisent si bien cette complémentarité que la traductologie pourrait bien devenir un lieu de rencontre stratégique entre linguistique et littérature.

L'étude des œuvres littéraires doit remonter des textes vers leur élaboration : c'est là une tâche majeure de l'herméneutique matérielle ou philologique. Elle s'articule en deux moments.

\footnotetext{
3 Je reprends en un autre sens ce néologisme de Rimbaud.

${ }^{4}$ Ne pouvant développer ici ce point, je me permets de renvoyer l'auteur, 2016 b.
} 
(i) D'une part, les œuvres éclairent les textes et l'on peut reconnaître que l'opératique, l'étude des œuvres, permet de discerner des opérations linguistiques non répertoriées. Sans prétendre que le langage dit quotidien n'est qu'une sécularisation du langage des dieux, du moins ses répétitions constantes évoquent-elles des ritualisations formulaires qui sous-tendent l'ordre social dans ses institutions différenciées.

(ii) D'autre part, les textes rendent compte de l'élaboration des unités inférieures, par répétition, figement, attrition, amuïssement. Un mot n'est pour ainsi dire qu'un fragment de mythe. Souligner cela n'est pas reprendre pour autant la thèse de Vico d'une origine poétique du langage : il n'a pas d'origine, car il est pris dans des évolutions, tant l'évolution darwinienne de notre espèce, qui a développé ses substrats anatomiques, que dans l'évolution lamarkienne de nos sociétés, qui détermine notre épigénèse et par là ce que l'on pourrait nommer la somatisation de la transmission sémiotique.

Il faut rappeler enfin, en opposition au principe frégéen de compositionalité, que l'on ne passe pas du simple au complexe ; dans les sciences de la culture, c'est la prise en considération de la complexité qui permet de déterminer les grandeurs et les traits pertinents.

\section{Directions et agenda}

Si les études littéraires rompaient avec les recherches scientifiques sur les langues et les textes, cela les mettrait dans la situation peu enviable d'une musicologie sans acoustique et sans organologie, voire d'une philosophie de la nature qui ignorerait tout de la physique.

Pour sa part, la linguistique a besoin d'ambition et l'intérêt qu'elle suscitait naguère a décru à proportion de sa dispersion en modèles partiels. Elle a tout intérêt à relever les défis multiples que lance la littérature, au niveau d'analyse qui est le sien et sans pouvoir prétendre supplanter les disciplines proprement littéraires ; par exemple, les tâches de caractérisation, comme celle de distinguer clairement Madame Bovary et Bouvard et Pécuchet, tâches banalement fondamentales pour les études littéraires, restent sous-estimées en linguistique. Voici quatre de ces défis.

(i) Renouer avec la philologie. - Comme le mot ou la phrase isolés ne sont que des artéfacts, le défi philologique doit être relevé, car les textes oraux et écrits sont l'objet empirique de la linguistique mais aussi son objet de connaissance. La philologie rappelle que rien n'est insignifiant, tout détail compte. Lors d'un atelier de lecture avec un ami, un différend s'éleva entre nous sur une fable de La Fontaine : après enquête, une « infime » différence de ponctuation dans nos éditions respectives suffisait à bouleverser la structure narrative. Cependant, aucune gram- 
maire formelle ne souffle mot de la ponctuation, qui en tout texte compte pourtant pour le cinquième des chaînes de caractères. Avec les méthodes d'enregistrement, la philologie s'est de fait étendue à l'oral, et l'essor de la linguistique de corpus a accompagné la constitution d'une philologie numérique (l'auteur 2001 : ch. 2).

(ii) Repenser la sémiosis. - La langue n'est pas un répertoire de signes déjà porteurs de leur sens, comme l'atteste l'insoluble question de la polysémie, faussée d'emblée par l'image lexicographique de la langue. La sémiosis n'est pas codée dans la langue. Si la langue propose voire impose des conditions toujours nécessaires, elles ne sont jamais suffisantes pour réaliser la sémiosis, constitution singulière et toujours renouvelée de signes en contexte - contexte qui s'étend au texte et même au corpus.

Les particularités dites « formelles » de la poésie, tout particulièrement, la rime, le mètre, le vers, la strophe, sont autant de moyens d'innovations sémiotiques. Cela s'étend à toute littérature « à contraintes »- elles le sont toutes, car une littérature devient créative à proportion des contraintes éthiques et esthétiques qu'elle s'impose. La qualité majeure de l'écrivain n'est pas l'imagination, substitut romantique de l'antique inspiration, mais, en fonction d'un projet éthique et esthétique, la capacité à tirer parti des moindres incidents du matériau linguistique pour en tirer des formes toujours renouvelées et toujours imprévues.

L'expérimentation sémiotique propre à la littérature recèle des enseignements majeurs. De même que les peintres conservent une avance sur les théoriciens de l'optique, les écrivains distancent souvent les linguistes, pour qui les traits de facture des œuvres sont ou devraient être des observables révélateurs. Les prendre en considération peut permettre d'approfondir la réflexion sur sémiosis, et de contribuer à une refondation de la linguistique. La sémiotique fut certes, dans le projet saussurien, une extension de la linguistique ; mais à mesure de cette extension, il faut à présent ménager en retour une reconception sémiotique de la linguistique, qui ne peut se satisfaire de l'apocryphe signe « saussurien » du Cours de linguistique générale et doit reconnaître l'hétérogénéité sémiotique des langues et des textes (l'auteur 2015). Pour cette reconception, l'étude des arts du langage revêt une évidente importance.

(iii) Tenir compte de l'interaction des langues. - La littérature permet tout à la fois d'étendre et de relativiser le concept de langue. Les nationalismes de jadis s'efforçaient de les enserrer dans des frontières, d'en faire l'expression de la « vision du monde » d'un peuple, quitte à les normer jusqu'à leur inventer une origine illusoire.

Or, les langues ne sont aucunement des isolats et les phénomènes de diffusion relevés par la linguistique aréale intéressent tous leurs niveaux d'analyse, car toute œuvre est hantée par d'autres, qu'elle remanie des originaux ou des traductions — qui d'ailleurs font œuvre elles aussi et contribuent à renouveler la langue. À présent, l'universalisme des grammaires formelles devrait être périmé par le 
cosmopolitisme raisonné qui présida à la fondation des sciences de la culture. L'exemple de la littérature engage à réaffirmer le principe comparatiste qui a pourvu la linguistique de sa base empirique et lui a permis de s'émanciper, de manière hélas non définitive, de préjugés millénaires de la philosophie du langage.

(iv) Problématiser l'interprétation. — La sémiosis établie, son interprétation peut être conçue comme une reconnaissance de formes. Comme la répétition est impossible, tout emploi d'un mot est un hapax, puisque chaque contexte, si prévisible soit-il, est nouveau. L'interprétation requalifie donc chaque signe à nouveau frais, sans d'ailleurs pouvoir toujours se stabiliser. Elle se trouve prise ainsi dans le cours d'une sémiosis illimitée a priori.

En 1956, Chomsky posait que l'interprétation n'est que le décalque de la génération et l'excluait de fait, suivi en cela par plusieurs générations de linguistes. Cependant, tous les textes, les œuvres littéraires au premier chef, posent des problèmes majeurs d'interprétation, qui ne peuvent aucunement être résolus par la compositionalité logico-syntaxique. La linguistique de corpus, l'émergence du web multilingue, tout cela engage à étendre le domaine de ce que Schleiermacher nommait déjà l'herméneutique matérielle, herméneutique non littéraliste mais qui tient strictement compte de la lettre.

Il importe aujourd'hui de substituer à la dualité entre grammaire et philosophie du langage, la dualité entre la linguistique générale et comparée et la philosophie des formes symboliques. Le projet complémentaire d'une sémiologie générale chez Saussure et d'une philosophie des formes symboliques, chez Cassirer, peuvent, après un siècle d'essor des recherches structurales et morphologiques, s'articuler dans une sémiotique des cultures conçue comme une théorie descriptive des institutions symboliques (langage, art, droit, technique, science, etc.). La liste de ces institutions est ouverte et leur articulation respective varie avec les cultures.

La littérature permet de découvrir la langue, décèle ses pouvoirs : les différences de tons, de registres, d'idiomatismes, de lexique, ouvrent toutes ensemble un monde d'effets de sens inconnus dans les autres usages, ordinaires ou non, auxquels un cours de langue ne saurait se limiter sans renoncer à stimuler la curiosité voire le désir d'apprendre.

Outre l'ouverture à cette diversité interne de la langue, l'étude de la littérature laisse entrevoir la diversité des langues, car les œuvres dialoguent sans cesse dans l'intertexte multilingue de la littérature internationale et font discrètement de leur lecteur un citoyen du monde, ce qui n'est pas sans intérêt dans publics divisés par des courants identitaires.

Il y a là un enjeu émancipateur, voire démocratique. La liberté prise par l'artiste permet à l'œuvre de faire événement et de continuer à trancher sur la grisaille du train-train quotidien. Elle peut devenir ainsi un exemple libérateur à l'égard des préjugés qui embrument tous les esprits. Comme les autres sciences 
et les autres arts, la linguistique et la littérature partagent en effet, chacune dans leur domaine, une dimension critique à l'égard des stéréotypes et des préjugés. Dans des conditions nouvelles, nous retrouvons alors le projet des Lumières, de l'Encyclopédie jusqu'aux sciences de la culture aujourd'hui : plus encore que de réconcilier deux cultures académiques, scientifique et littéraire, jadis abusivement séparées, il faut réconcilier la culture mondiale avec elle-même, au-delà des idéologies identitaires et des replis nationalistes.

N.B. — Les présentes réflexions s'appuient sur divers ouvrages d'analyse littéraire (l'auteur : 2005, 2016a, 2018, 2019) impossibles à évoquer ici dans les sages limites imparties.

\section{Références bibliographiques}

Chomsky 1966 : N. Chomsky, La linguistique cartésienne, Paris : Seuil.

Chomsky 1984 : N. Chomsky, La connaissance du langage, Communications, 40, p. 7-34. Glissant 1975 : É. Glissant, Malemort, Paris : Gallimard.

Havet 1922 : L. Havet, Célébration du cinquantenaire de l'Ecole pratique des hautes études, Paris : Champion.

La Grasserie 1893 : R. de La Grasserie, De la classification objective et subjective des arts, des sciences et des lettres, Paris : Alcan.

Rastier 1987 : F. Rastier, Sémantique interprétative, Paris : PUF.

Rastier 2005 : F. Rastier, Ulysse à Auschwitz. Primo Levi, le survivant, Paris : Cerf.

Rastier 2016a : F. Rastier, Créer : image, langage, virtuel, Paris-Madrid, Casimiro.

Rastier 2016b : F. Rastier, Sens et textualité, Limoges : Lambert Lucas (deuxième édition augmentée).

Rastier 2015 : F. Rastier, Saussure au futur, Paris : Les Belles-Lettres/Encre marine.

Rastier 2018 : F. Rastier, Mondes à l'envers. De Chamfort à Samuel Beckett, Paris : Garnier.

Rastier 2019 : F. Rastier, Témoignages inconcevables. Littératures et exterminations, Paris : PUF (sous presse). 
Франсоа Растје

\section{ЛИНГВИСТИКА И НАУКА О КЫИЖЕВНОСТИ}

Иако опис књижевности не припада граматичкој традицији, још од настанка лингвистике, пре два века, наука о језику и наука о књижевности сусрећу се на подручју наука̂ о култури. Њихов сусрет је, међутим, остајао у сенци формалних граматика и ортодоксног когнитивизма. Ипак, семиотика култура̂ ослањала се на теоретска достигнућа настала захваљујући развоју упоредне методе у оквиру структурализма. Стога је, надилазећи лингвистику текста, могуће осмислити и у истраживања укључити и лингвистику дела. Лингвистика и књижевна студије могу заиста много научити из својих интеракција.

Кључне речи: упоредна лингвистика, књижевност, структурализам, граматика, семиоза, семиотика култура̂ 